\title{
Tests of the Gravitational Inverse Square Law at Short Ranges
}

\author{
R.D. Newman • E.C. Berg • P.E. Boynton
}

Received: 16 March 2009 / Accepted: 12 May 2009 / Published online: 4 June 2009

(C) The Author(s) 2009

\begin{abstract}
A laboratory test and several geophysical tests conducted in the last decades of the 20th century suggested deviations from the inverse square distance dependence of Newton's law of gravity. While further work has failed to substantiate these results, renewed interest in inverse square law tests of increased sensitivity has been stimulated by a wide range of new theoretical ideas. Of particular interest are tests at submillimeter ranges, which could reveal the existence of compact new dimensions accessible only to gravity. This paper reviews the current status of inverse square law tests, with emphasis on present and proposed experimental techniques.
\end{abstract}

Keywords Inverse square law $\cdot$ Newton's law of gravity

\section{Introduction}

In 1974 Daniel Long published a paper Why do we believe Newtonian gravitation at laboratory dimensions? (Long 1974), comparing measurements of $G$ made since 1894 with various mass separations $r$. His plot of $G$ values as a function of $r$ strongly suggested a dependence on mass separation. Two years later, Long reported an experiment of his own (Long 1976) which used a torsion balance to compare the forces produced by source masses at distances of $4.5 \mathrm{~cm}$ and $29.9 \mathrm{~cm}$. Long's experiment used ring-shaped source masses, exploiting the fact that the force on a test mass at a certain point on the axis of a ring source mass is at an extremum and thus is quite insensitive to error in its position relative to the ring. Daniel Long reported that the ratio of the torque produced by the more distant ring to that produced by the nearer ring exceeded the Newtonian prediction by $(0.37 \pm 0.07) \%$, a result consistent with the distance dependence found in his analysis of $G$ measurements.

R.D. Newman $(\bowtie) \cdot$ E.C. Berg

Department of Physics and Astronomy, University of California, Irvine, CA 92697, USA

e-mail: rdnewman@uci.edu

P.E. Boynton

Department of Physics, University of Washington, Seattle, WA 98195, USA 
This result precipitated a flurry of efforts by others to search for inverse square law violation (ISLV). The early ISLV tests probed ranges greater than one millimeter, and quite conclusively ruled out the violation apparently found by Long. More recently attention has focused on searches for ISLV at sub-millimeter ranges, motivated by a wide variety of theoretical speculations. An excellent review of the 2003 status of these searches and their theoretical motivations has been presented by Adelberger et al. (2003). A recent paper by Adelberger and co-authors (Adelberger et al. 2009) updates this review for tests conducted with torsion balances. Here we review the status of ISLV tests with emphasis on progress since 2003, prospects for improved tests, and the principles of instrumentation employed in this area of research.

Detecting Anomalous Forces A natural assumption is that a new force in nature or an anomaly in classical gravity can be characterized by an interaction of Yukawa form:

$$
U(r)=\frac{-G m_{1} m_{2}}{r}\left(1+\alpha e^{-r / \lambda}\right)
$$

with strength $\alpha$ relative to Newtonian gravity, and range $\lambda=\frac{\hbar}{m c}$ related to the mass of a mediating particle. A power law dependence could also naturally arise in some cases, and is considered briefly later in this review. Here we assume an interaction of Yukawa form (1). The strength parameter $\alpha$ may be a function of the composition of the interacting masses, leading to a real (or apparent) violation of Einstein's Weak Equivalence Principle. There are then two complementary approaches to the search for the anomalous interaction:

1. One may search directly for deviation from inverse square law behavior. This has the advantage that it works even if composition dependence is very weak or nonexistent. It's disadvantage is that for useful sensitivity the scale of the experiment needs to be on the order of $\lambda$. This is evident from the force law following from (1):

$$
F(r)=\frac{G m_{1} m_{2}}{r^{2}}\left(1+\alpha\left(1+\frac{r}{\lambda}\right) e^{-r / \lambda}\right)=\frac{G(r) m_{1} m_{2}}{r^{2}}
$$

where the anomalous $r$ dependence is absorbed in an effective $G(r)$. A plot (Fig. 1) of $G(r)$ shows that to have optimum sensitivity an ISLV test must compare forces at ranges roughly straddling $\lambda$. Thus a thorough test for inverse square law violation requires an extensive set of experiments, each tailored for a different region in $\lambda$ parameter space.

2. Alternatively, one may search for composition dependence of the interaction strength $\alpha$. This has the advantage of retaining sensitivity for all mass separations not much greater than $\lambda$, but suffers from the fact that the difference in composition of suitable test bodies (by such measures as neutron/proton ratio or baryon number/mass ratio) is generally very small. Thus the two approaches are complementary and ideally are both to be pursued.

\section{Laboratory ISLV Tests at Ranges Greater than a Millimeter}

The period between Daniel Long's (1976) paper and 1993 yielded many searches for ISLV testing ranges from a few millimeters to a few meters. Current constraints in $\alpha-\lambda$ parameter space established by the more sensitive tests are displayed in Fig. 2, along with the region of parameter space allowed by Long's positive result.

The first experiment (Spero et al. 1980) to apparently exclude Long's effect yielded the constraint labeled "Irvine I" in Fig. 2. This was a "near null" experiment (Fig. 3) conducted 
Fig. 1 Plot of $G(r)$ given by (2) for $\alpha=0.2$, demonstrating that detection of inverse square law violation by comparing forces at two mass separations requires that the chosen separations roughly flank $\lambda$
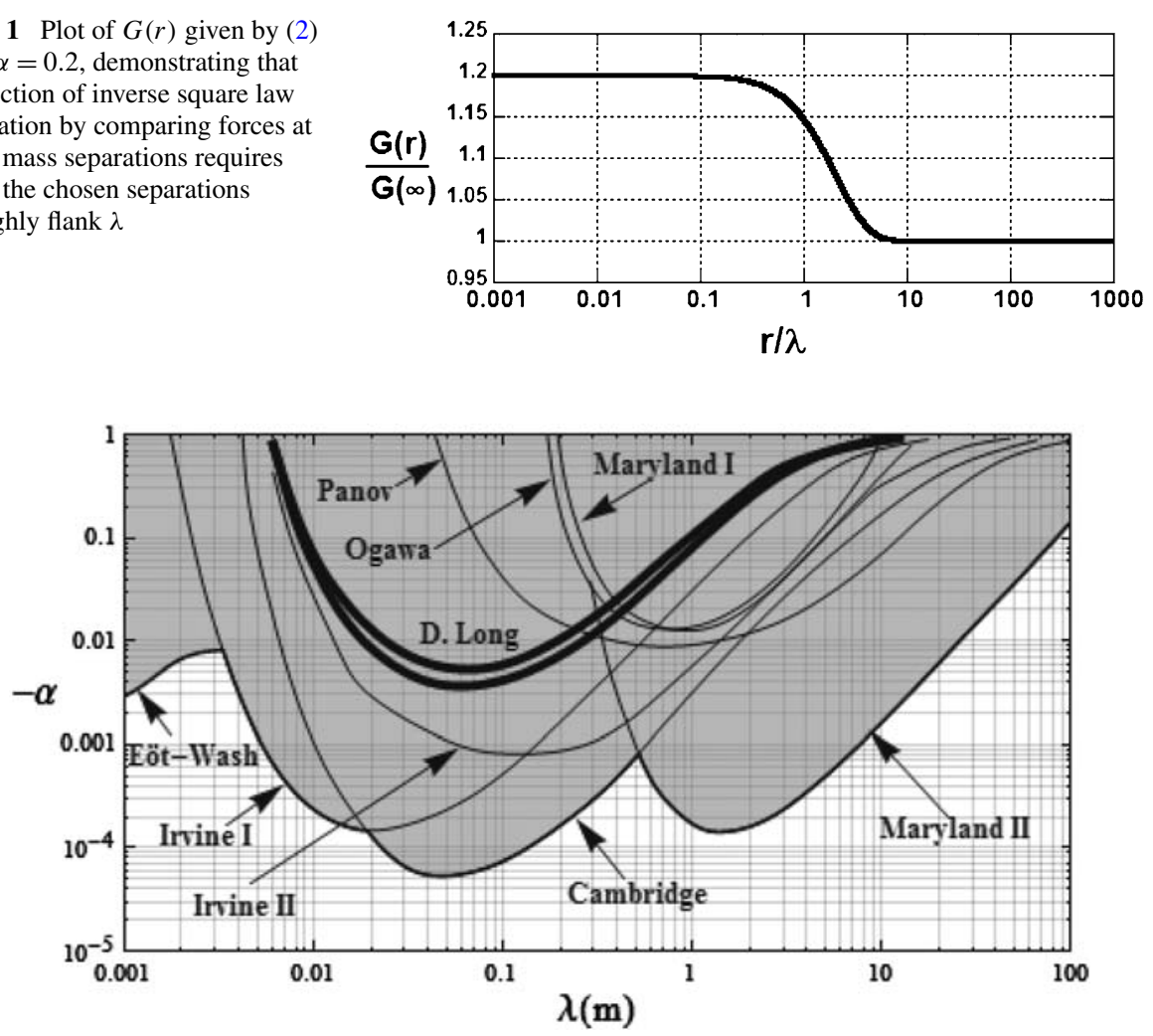

Fig. 2 Experimental limits on an interaction of the form of (1) with $\lambda>1 \mathrm{~mm}$ and negative $\alpha$, with the $1 \sigma$ limits (dark lines) of the parameter space implied by Long's experiment (Long 1976). Other lines are $1 \sigma$ limits, except the Eöt-Wash line which is a $2 \sigma$ limit on $|\alpha|$. References are as follows: Irvine I and II: (Spero et al. 1980; Hoskins et al. 1985), Eöt-Wash: (Kapner et al. 2007), Cambridge: (Chen et al. 1984), Maryland I and II: (Chan et al. 1982; Moody and Paik 1993), Panov: (Panov and Frontov 1979), Ogawa: (Ogawa et al. 1982). A corresponding plot for positive $\alpha$ is presented in Fig. 6

at UC Irvine, which exploited the fact that for an inverse square force law the gravitational $\mathrm{g}$ field vanishes within an infinite mass tube just as within a spherical mass shell. The suspended test mass was used to probe the change in gravitational field near the center of a stainless steel tube as the tube was moved laterally. The small $(\approx 1 \%)$ end effect from the finite length of the tube was largely cancelled by a thin copper ring that was moved from one side of the test mass to the other as the tube was translated.

Daniel Long then suggested (Long 1980) that the anomaly he found might arise from a vacuum polarization effect analogous to that producing a logarithmic deviation from $1 / r^{2}$ behavior in the electric force between charges at very short distances. Such an effect might not be observable in a null experiment such as the Irvine test, Long argued, because of the lack of a polarizing field in the region probed by the test mass. However, a number of subsequent experiments that were not of null type, including one at Irvine (Hoskins et al. 1985), gave results consistent with Newton's law but not with the violation reported by Long. It is likely that Long's experiment suffered from an undetected systematic error from apparatus tilt correlated with source mass position —an insidious and common problem in torsion pendulum gravity experiments. 
Fig. 3 Apparatus of the first Irvine ISLV test. A test mass probes the gravitational field within a SS tube of height $60 \mathrm{~cm}$ ID $6 \mathrm{~cm}$ and OD $8 \mathrm{~cm}$

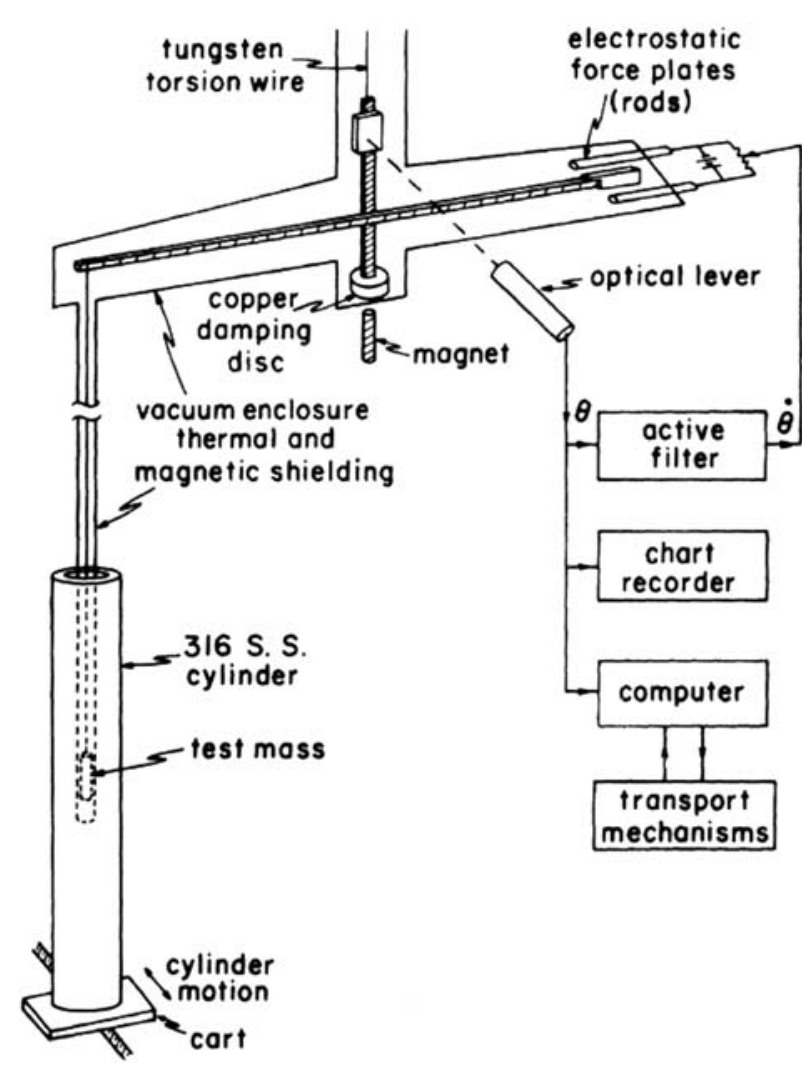

Laboratory tests of ISLV at ranges above a few millimeters have taken a number of interesting forms. Several have used a torsion pendulum (Chen et al. 1984; Panov and Frontov 1979; Spero et al. 1980; Hoskins et al. 1985). A test by Goodkind et al. (1993) at UC San Diego measured the change of force exerted on a levitated superconducting niobium ball when a spherical mass was placed below it at a distance ranging from 0.4 to 1.4 meters. A test by Ogawa et al. (1982) at the University of Tokyo measured the resonant response of a $1400 \mathrm{~kg}$ aluminum quadrupole gravity wave antenna to a $401 \mathrm{~kg}$ steel bar rotating at $30.4 \mathrm{~Hz}$ and placed at distances ranging from 2.6 to 10.6 meters. A test by Moody and Paik (1993) at the University of Maryland used a three-axis superconducting gradiometer to detect a possible non-zero Laplacian of the gravitational field generated by a $1500 \mathrm{~kg}$ lead pendulum (Fig. 4). As the Laplacian of the Newtonian gravitational field $\left(\nabla^{2} \Phi\right)$ must vanish, this was an elegant null experiment. Each axis of the gradiometer instrument was a pair of superconducting accelerometers read out by a differential SQUID circuit-a technique that Paik is now applying to a sub-mm ISLV test described here in Sect. 3.

Finally, we describe a torsion pendulum ISLV test being conducted by the authors together with Michael Moore and Ricco Bonicalzi in a U. Washington/UC Irvine (UW/UCI) collaboration. This experiment (Boynton et al. 2007) is designed to have maximum sensitivity in $\alpha-\lambda$ parameter space at $\lambda \sim 12 \mathrm{~cm}$. The test, like that of Moody and Paik (1993), exploits the fact that ISLV is signaled by a non-vanishing Laplacian of the gravitational field. In our test, the signal is the gradient of the Laplacian, manifest as an $m=1$ variation in the pendulum's torsional frequency and anharmonic behavior as a source mass re- 


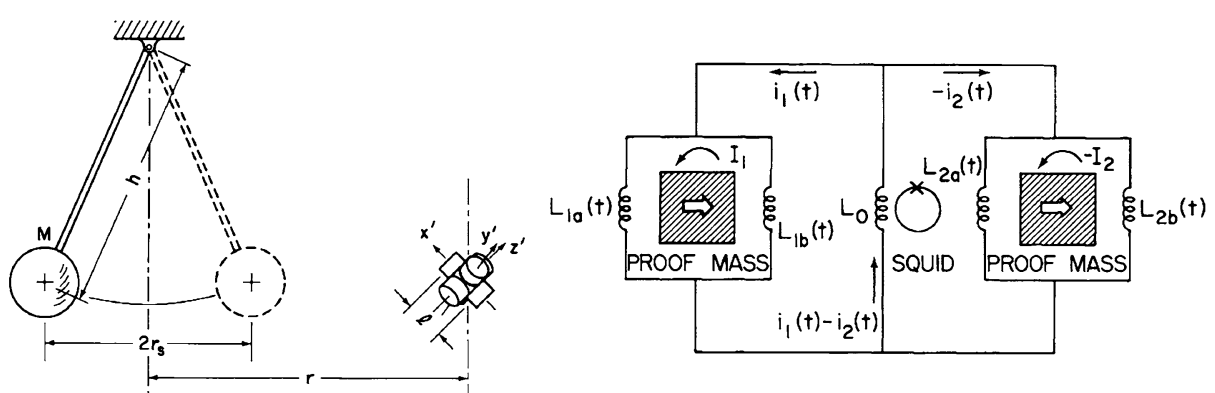

Fig. 4 Principle of Ho Jung Paik's (1993) inverse square law test. Signals from a cryogenic three-axis gradiometer (central figure) are summed to yield the Laplacian of the gravitational field $\Phi$ generated by a swinging pendulum. Each of the three orthogonal gradiometers $(i=1,2,3)$ uses the circuit shown to measure the differential displacement of two test masses separated by a distance $\ell$. This yields the differential force $\Delta F_{i}$ on the test masses $m$, and thus yields $\partial g_{i} / \partial x_{i}=\Delta F_{i} /(m \Delta \ell)$ which are summed to yield $\nabla^{2} \Phi$

volves around the pendulum in a sequence of stationary positions. The experiment has two key design features: a pendulum that couples to the gradient of the Laplacian, but has no $m=1$ mass moments that couple to Newtonian gravitational fields through $\ell=6$, and a source mass configuration that would exaggerate the gradient of the Laplacian $\left(\nabla \nabla^{2} \Phi\right)$ of a Yukawa potential, but generates no $m=1$ Newtonian field moments through $\ell=8$. Either of these design features, if achieved, would suffice to eliminate significant bias from Newtonian field couplings in this experiment. The combination of these features renders the experiment extremely insensitive to small fabrication errors in either pendulum or source mass. The experiment's pendulum and source mass are shown in Fig. 5.

The experimental design and analysis (Moore 2000) generalizes the standard multipole formalism to allow for non-Newtonian fields such as $V(r) \propto \frac{1}{r} e^{-r / \lambda}$. One finds that the general interaction energy $U=\int \rho V d^{3} r$ between a pendulum of density distribution $\rho(r)$ and the gravitational field $V$ produced by a source mass may be expanded:

$$
U=\sum_{n \ell m} V_{n \ell m} M_{n \ell m}
$$

where $M_{n \ell m}$ is the generalized multipole mass moment of the pendulum

$$
M_{n \ell m} \propto \int \rho r^{n} Y_{\ell m} d^{3} r
$$

and $V_{n \ell m}$ is constructed from $n$ th-order derivatives of the field $V$ at the origin of the pendulum. This generalized multipole formalism includes non-Newtonian multipole terms for which $\ell$ is smaller than $\mathrm{n}$ by increments of 2 .

The horizontal gradient of the Laplacian would manifest itself specifically in the multipole term

$$
U_{311}=V_{311} M_{311} \propto \frac{\partial}{\partial x}\left(\nabla^{2} V\right) \int \rho r^{3} Y_{11} d x d y d z
$$

This experiment is hosted by the Pacific Northwest National Laboratory, and operates in a former Nike missile bunker on a remote arid lands preserve near Richland, Washington. The initial phase of this work is conducted at room temperature, using the second harmonic amplitude of the pendulum's oscillation as a measure of the ISL-violating torque that would 

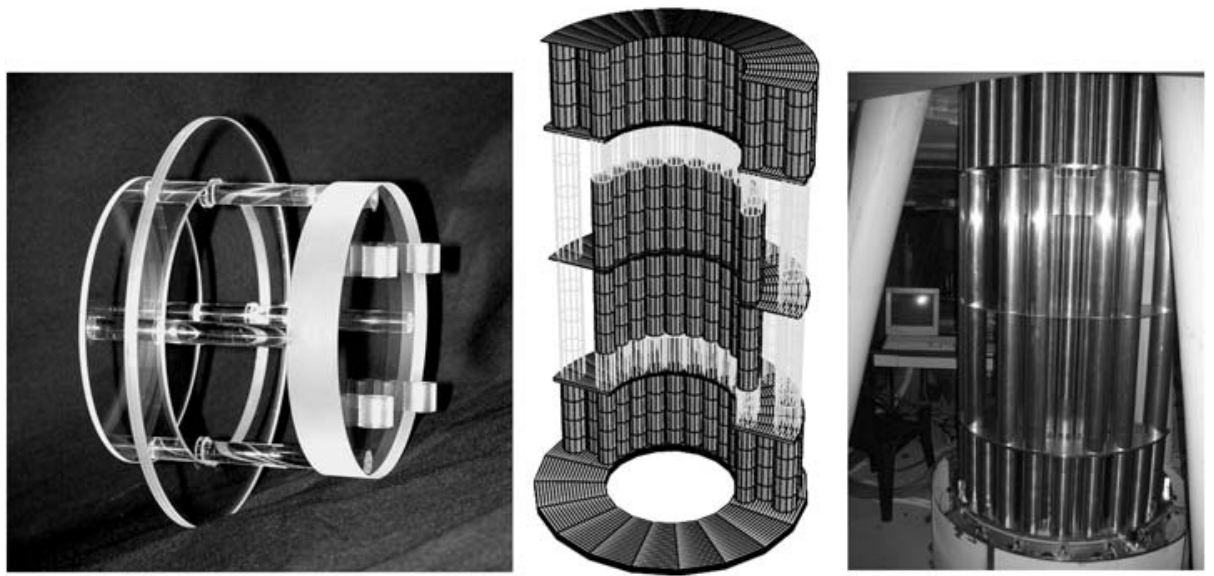

Fig. 5 The pendulum and source mass of the UW/UCI ISLV test. The pendulum, shown before gold coating, is made of fused silica and has a diameter of $11.5 \mathrm{~cm}$. Not shown are a set of four small gold-plated titanium trim masses. The 1.4 tonne $1.6 \mathrm{~m}$ high source mass, constructed from stainless steel solid cylinders and hollow spacer tubes, is shown as a rendition and as realized. The source mass rotates at intervals on an air bearing base. Various alternate mass stacking configurations may be constructed to deliberately exaggerate Newtonian field gradients for diagnostic purposes

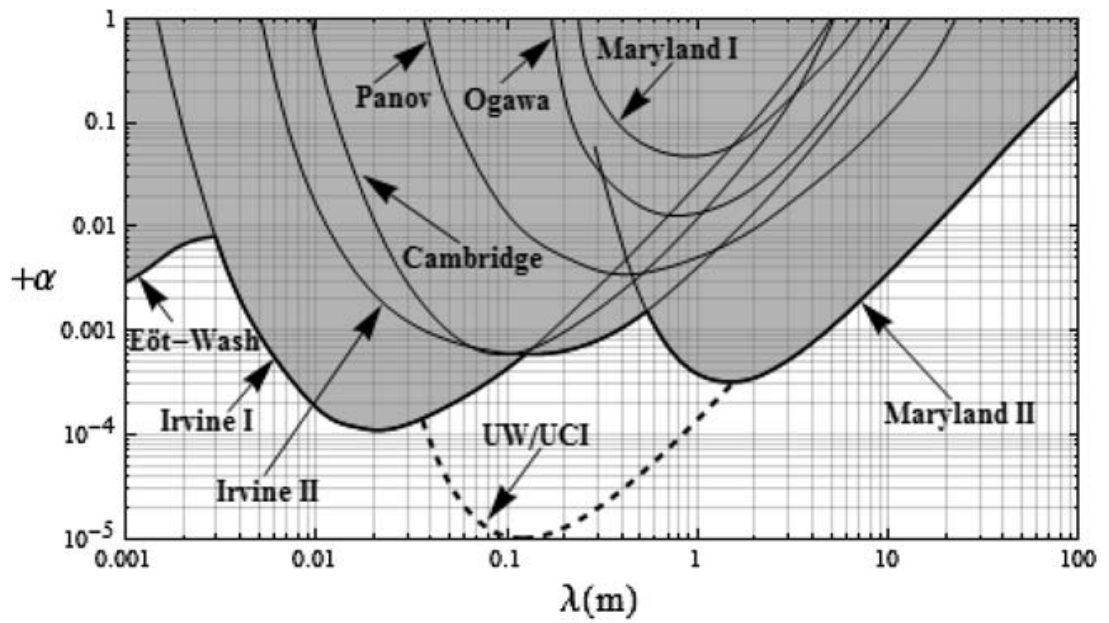

Fig. 6 Experimental limits on an interaction of the form of (1) with $\lambda>1 \mathrm{~mm}$ and positive $\alpha$. Limits shown are $1 \sigma$, except the Eöt-Wash line which is a $2 \sigma$ limit on $|\alpha|$. Plot labels correspond to references as in Fig. 2. The dashed line indicates the projected sensitivity of the UW/UCI experiment in progress. A corresponding plot for negative $\alpha$ is presented in Fig. 2

arise from $U_{311}$. In the second experiment phase, the pendulum is in a cryogenic environment at $\sim 4 \mathrm{~K}$, using the variation of torsion frequency as a measure of $U_{311}$. A $1 \sigma$ sensitivity at a level $\alpha=10^{-5}$ is anticipated, with the projected constraint in $\alpha-\lambda$ parameter space indicated in Fig. 6. 
Fig. 7 Scale drawing of the Eöt-Wash instrument. The three spheres near the top of the detector served for a gravitational calibration of the torque scale. The detector's electrical shield is not shown

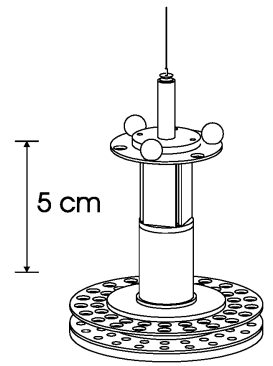

\section{ISLV Tests from $1 \mu \mathrm{m}$ to $1 \mathrm{~mm}$}

Published constraints in $\alpha-\lambda$ parameter space and projected improvement in constraints from the experiments discussed in this section are displayed in Figs. 13 and 14.

\subsection{Torsion Pendulum Sub-mm Experiments}

The University of Washington "Eöt-Wash" group is conducting a series of ISLV tests (Hoyle et al. 2001, 2004; Kapner et al. 2007). The most recent of these tests probed mass separations ranging from $9.53 \mathrm{~mm}$ to $55 \mu \mathrm{m}$. These place the best current limits on ISLV below about $3 \mathrm{~mm}$, and put a 95\% confidence limit of unity on the magnitude of $\alpha$ (the strength of a Yukawa term relative to gravity, (1)) down to a length scale $\lambda=56 \mu \mathrm{m}$. The instrument used (Fig. 7) was a torsion pendulum detector suspended above an "attractor" which rotated at a constant velocity $\omega$ lower than the pendulum's resonant frequency by a factor of 28 or 7.5. The detector's 42 "negative mass" test bodies were 5-mm-diameter holes in a 1-mmthick molybdenum detector ring. The attractor was a 1-mm-thick molybdenum disk with 42 3.2-mm-diameter holes mounted above a thicker tantalum disk containing 21 6.4-mmdiameter holes. A gold-coated $10-\mu$ m-thick $\mathrm{CuBe}$ shield was stretched between the attractor and pendulum to block electrostatic interaction. The gravitational interaction between the missing masses of attractor and detector holes produced a torque with harmonics at multiples of $21 \omega$, with resulting angular displacement read out by an autocollimator. The two disks of the attractor were displaced azimuthally and were designed to nearly cancel the $21 \omega$ signal in the absence of ISLV. Capacitive and micrometer techniques served to measure relative position and parallelism of the attractor and pendulum disks. An important advantage of the experiment's multi-hole design is that the signal frequency is far removed from frequencies of possible periodic disturbances. Experiments currently in progress by this group include one using a variant of the apparatus shown in Fig. 7 having 120 radial wedge-shaped $100 \mu \mathrm{m}$ wide holes, and one using a torsion pendulum with flat plates suspended in a vertical plane near a plane source mass.

Jun Luo's Huazhong University group in Wuhan China (Tu et al. 2007) used a torsion pendulum (Fig. 8) to probe the force between a planar gold source and test masses for mass separations from 176 to $341 \mu \mathrm{m}$, putting a $95 \%$ confidence limit on a gravitational-strength $(|\alpha|=1)$ interaction down to a length scale $\lambda=66 \mu \mathrm{m}$. Between source and test masses was a $56 \mu \mathrm{m}$ glass membrane with a 300 -nm gold coating on each side. Like the Eöt-Wash instrument, the Wuhan instrument is designed to give a nearly null Newtonian signal over the range of separations explored.

Ramanath Cowsik at Washington University in Saint Louis is developing an ISLV test in which a torsion ribbon suspends a 1-mm-thick 100-mm-diameter metal disk in a vertical 


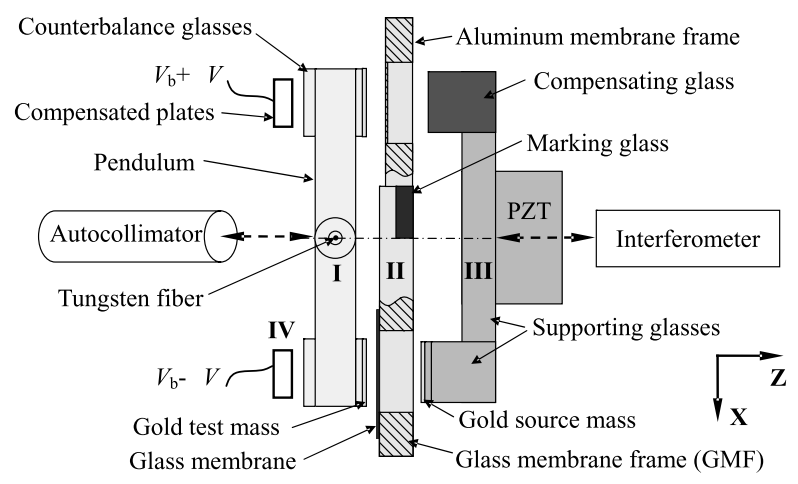

Fig. 8 Schematic top view of the Wuhan apparatus, showing: (I) the pendulum, carrying the gold test mass and a counterbalance mass, (II) a frame carrying gold-coated electrostatic shield membranes, (III) a PZT-driven moving frame carrying the gold source mass with a compensating mass to largely null the Newtonian interaction, and (IV) electrostatic force plates to null torsional displacement. Translation of element III and rotation of the pendulum I are read by the indicated interferometer and autocollimator
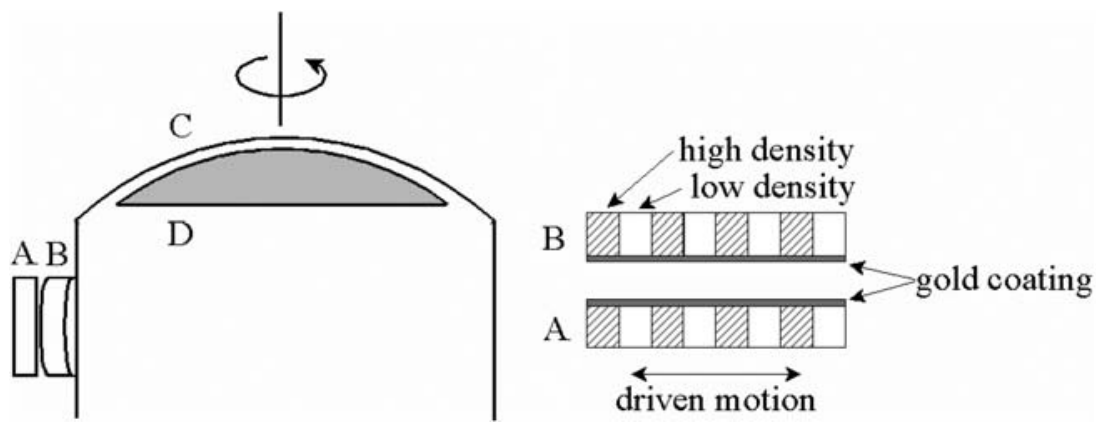

Fig. 9 Schematic cross-section view of the Birmingham superconducting-suspension torsion balance applied to a sub-mm ISLV test. A superconducting niobium spherical-surface cap (C) is levitated by current in a spiral niobium wire winding embedded in a MACOR spherical-surface bearing (D) of $43 \mathrm{~mm}$ radius. Mounted on the levitated "balance" is an array (B) of metal bars of alternately high and low density. A similar array (A) is driven with frequency $\omega$ in the indicated direction, producing a torque on the levitated balance at frequency multiples of $\omega$. The resulting angular displacement of the balance is read out with a capacitive or SQUID-based technique. Mass arrays A and B each have a gold coating, but no intervening shield

plane, parallel to a much larger diameter iridium source mass disk. Between the disks is a thin electrostatic shield. The position and angular orientation of the source mass disk are to be independently modulated at carefully selected frequencies, thereby modulating both the mean and differential distance between the source disk and regions of the test mass on opposite sides of the torsion ribbon. Cowsik has developed a remarkably lownoise autocollimator $\left(\approx 2 \times 10^{-10} \mathrm{rad} / \sqrt{\mathrm{Hz}}\right.$ at $\left.10 \mathrm{mHz}\right)$ for angular readout of the test mass pendulum.

Clive Speake and collaborators at the University of Birmingham are developing a sub-mm ISLV test based on a novel superconducting torsion balance (Hammond et al. 2008), illustrated schematically in Fig. 9. 


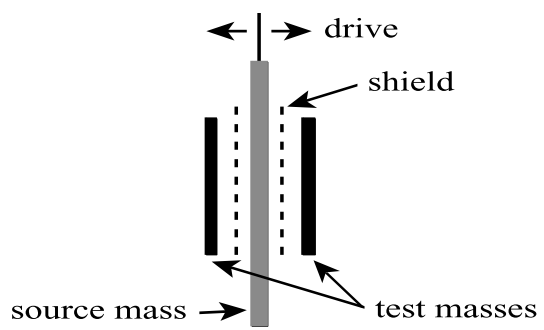

Fig. 10 Principle of the Maryland ISLV cryogenic instrument. The source mass disk is driven at frequency $\omega$ as shown. The spring-loaded test mass disks experience a differential force at $2 \omega$, which would be zero for a Newtonian force law and infinite disk diameter. The detected differential force, corrected for finite disk sizes, is thus a measure of ISLV. The differential displacement of the test masses is measured with a SQUID-based technique like that used in the gradiometers in the 1993 Maryland ISLV test at longer ranges (Fig. 4)

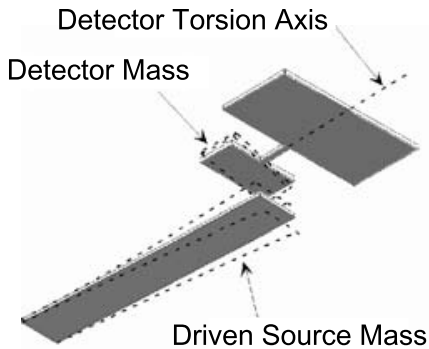

Source and Detector Oscillators

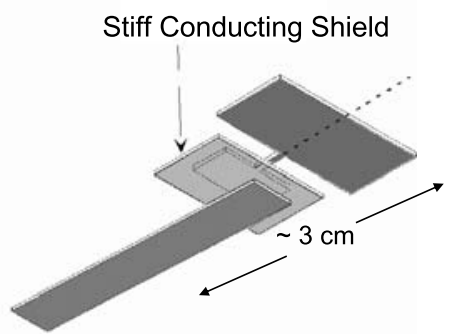

Shield for Background Suppression

Fig. 11 Principle of Josh Long's ISLV test. A PZT bimorph drives a tungsten source mass at the $\sim 1 \mathrm{kHz}$ resonant frequency of a tungsten double torsional oscillator that serves as test mass. A thin sapphire plate with $100 \mathrm{~nm}$ gold coating on both sides is interposed between source and test masses. A capacitive transducer measures the amplitude of the torsional oscillation. The source and test mass systems are mounted on independent vibration isolation stacks, each of which attenuates vibration by a factor of $10^{10}$ at $1 \mathrm{kHz}$

\subsection{Non-Torsion-Pendulum Sub-mm ISLV Tests}

Ho Jung Paik and collaborators at the University of Maryland are conducting a cryogenic ISLV test based on measurement of the differential motion of a pair of parallel circular test mass disks when an interposed source mass disk is moved away from one test mass and toward the other (Fig. 10).

Josh Long at Indiana University is continuing an ISLV test he developed with John Price at the University of Colorado (Long et al. 2003). This experiment measures the resonant response of a test-mass-carrying cantilever to a source mass driven up and down below it (Fig. 11).

Aharon Kapitulnik and collaborators at Stanford University have been conducting a series of ISLV tests of steadily increasing sensitivity using a test mass mounted on a resonant cantilever above an array of elements of alternating high and low density material moving below it. In most of these tests a short source mass array is moved back and forth by a PZT driver at a frequency which is a sub-multiple of the cantilever resonant frequency, causing the alternating source mass elements to drive the cantilever at resonance. In this way danger of a spurious signal from mechanical coupling of the drive mechanism to the detector is 


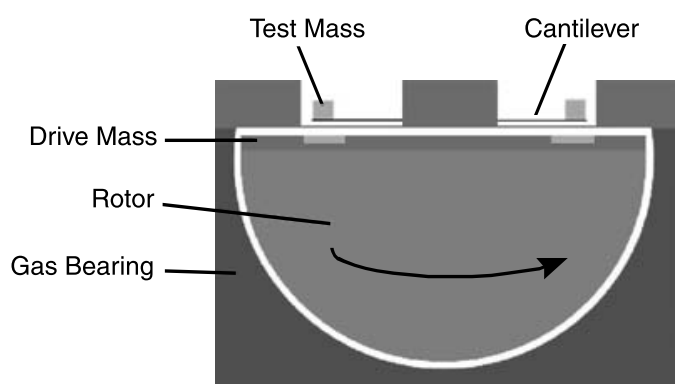

Fig. 12 Cross-sectional diagram (not to scale) of the Stanford ISLV apparatus of Weld et al. (2008). The indicated drive mass is a disk of low-density material (tungsten or brass) in which trenches have been etched or machined and filled with another, less-dense material for purposes of planarization, yielding a circular array of 100 "source masses". The drive mass is bonded to a $5 \mathrm{~cm}$ diameter cryogenic fused quartz helium gas bearing which rotates at about $3.5 \mathrm{~Hz}$. Above the source mass plane are test masses mounted on cantilever detectors resonant at about $350 \mathrm{~Hz}$, whose displacement is read out with fiber interferometers. On the top surface of the source mass array is a uniform flat coating of gold. A gold-coated silicon nitride shield membrane separates the rotating assembly from the test masses. The face-to-face separation between source and test masses can be as small as $29 \mu \mathrm{m}$, and may be accurately varied as much as $5 \mu \mathrm{m}$ by varying the bearing gas flow to raise or lower the spinning rotor

minimized. Preliminary results have been reported from a new experiment design in which a circular array of 100 alternating mass elements mounted on a cryogenic gas bearing passes continuously below the sensor cantilever (Fig. 12). This promising approach has a number of advantages, including ease of stable alignment and ability to employ relatively large area source masses. A problem has been vibrational noise generated by the gas bearing, which the researchers expect to reduce in future work.

\subsection{General Considerations for Very Short Range ISLV Tests}

Among the ISLV tests described above that explore mass separations below $1 \mathrm{~mm}$, most measure a force normal to the surface of parallel mass plates. This has the advantage that the full area of the mass plates contribute to the measured signal force. In contrast, the multihole torsion pendulum of the Eöt-Wash group and the spherical superconducting torsion balance of the Birmingham group measure a force tangential to planar arrays of facing mass elements as one plane moves laterally with respect to the other. This approach has the disadvantage that, for an anomalous force with range $\lambda$, only a portion of the interacting masses of width $\lambda$ on their leading and trailing edges contribute significantly to the measured signal. This is an increasing disadvantage as the force range probed becomes smaller. However, this disadvantage is largely compensated by the extraordinary mechanical compliance of a torsion fiber. It is interesting to compare instruments in terms of an effective "spring constant" relating force to displacement. The Eöt-Wash torsion pendulum has a $3 \times 10^{-9} \mathrm{Nm} / \mathrm{rad}$ torsion constant, or $2.4 \times 10^{-6} \mathrm{~N} / \mathrm{m}$ effective linear spring constant. This may be compared with Decca's MEMS torsion oscillator (following section, Fig. 15), which has a smaller torsion constant $\left(8.6 \times 10^{-10} \mathrm{Nm} / \mathrm{rad}\right)$, but because of its short $(0.2 \mathrm{~mm})$ lever arm has a linear spring constant $2.2 \times 10^{-4} \mathrm{~N} / \mathrm{m}$, two orders of magnitude stiffer. The Stanford cantilever has a linear spring constant of about $10^{-2} \mathrm{~N} / \mathrm{m}$, nearly four orders of magnitude greater than that of the torsion pendulum.

Gravitation ISLV tests must contend with competing magnetic, electrostatic, and Casimir forces. For tests at ranges greater than a few millimeters, electric and magnetic shielding is 


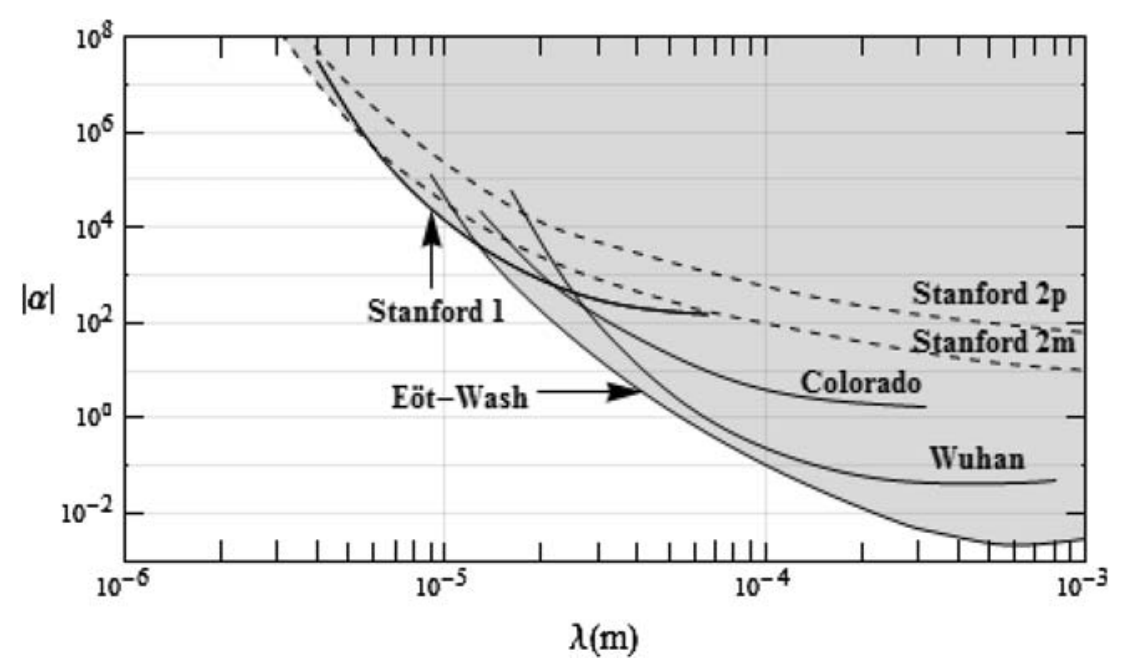

Fig. 13 Limits at 95\% confidence level, as of January 2009, on an anomalous interaction of Yukawa form with range between $\lambda \sim 1 \mu \mathrm{m}$ and $1 \mathrm{~mm}$. Curve labels and references correspond as follows: Stanford 1 (Geraci et al. 2008), Stanford 2p and 2m (Weld et al. 2008), Colorado (Long et al. 2003), Wuhan (Tu et al. 2007), Eöt-Wash (Kapner et al. 2007). Stanford 2 p and $2 \mathrm{~m}$ represent $|\alpha|$ limits assuming $\alpha$ greater or less than zero respectively

easily achieved, but at shorter ranges becomes difficult or impossible. Conventional wisdom is that Casimir forces are adequately shielded by a gold layer thicker than the $\sim 140 \mathrm{~nm}$ plasma wavelength of gold. Gold-coated shields as thin as $4 \mu \mathrm{m}$ have been employed between source and test masses, but care must be taken that a shield is sufficiently stiff that no distortion correlated with source mass position is generated by pressure, vibration, or electrostatic forces. One approach, illustrated in the Birmingham experiment, is to dispense with a free-standing shield and rely on "isoelectronic" facing surfaces that are either uniformly gold-coated or possibly made of differing isotopes of the same material. In either case the Casimir force is the same for source mass regions of different density, allowing a difference in gravitational attraction to be discerned. In this approach extraordinary care must be taken to ensure that the surface quality is identical for the differing density mass regions, and is extremely smooth.

Figure 13 shows current limits in $\alpha-\lambda$ parameter space on a new force of range $\sim 1 \mu \mathrm{m}$ to $1 \mathrm{~mm}$. Figure 14 shows targeted improved limits projected by various groups.

\section{ISLV Tests Below $\sim 1 \mu \mathrm{m}$}

To explore mass separations below a few microns one has little choice but to accept a distance-dependent Casimir force directly, extracting gravitational anomalies by comparing a measured force with the Casimir prediction. This is complicated by uncertainties in Casimir force calculation, associated with surface quality, temperature, etc. A recent publication (Mostepanenko et al. 2008) derives 95\% confidence limits on a new force in $\alpha-\lambda$ parameter space from several Casimir force experiments, see Fig. 17. The most recent such experiment (Decca et al. 2005), Fig. 15, used a MEMS torsion oscillator to measure the Casimir force between gold surfaces at separations from 160 to $750 \mathrm{~nm}$. This experiment 


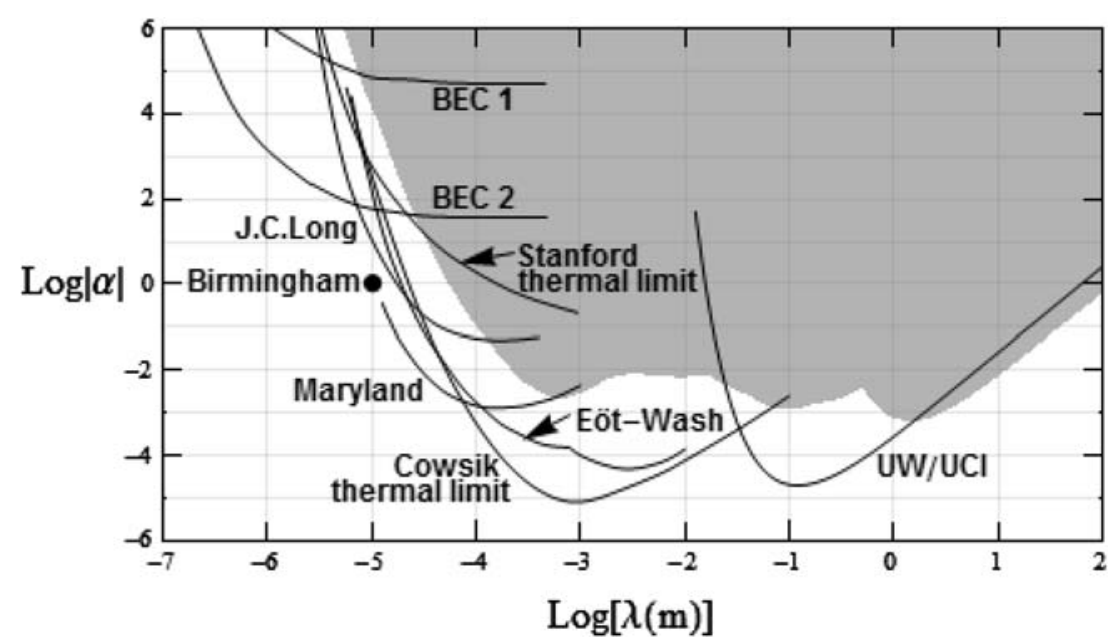

Fig. 14 Possible future sensitivities projected by various groups, for searches for an anomalous interaction of Yukawa form (1). Lines BEC 1 and 2 are from Dimopoulos and Geraci (2003), line Stanford is from Weld et al. (2008); the other projections derive from unpublished work

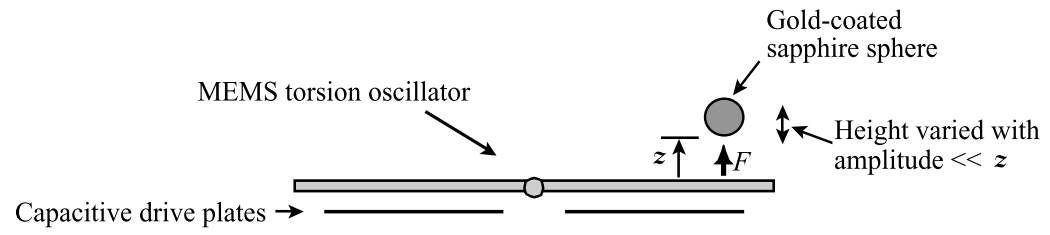

Torsion constant $k=k_{o}+c \frac{\partial F}{\partial z}$

Fig. 15 Principle of the Casimir measurements of Decca et al. (2005). A micromechanical torsion oscillator operates at $\sim 700 \mathrm{~Hz}$. At height $z$ above the gold-coated oscillator plate a 0.3 -mm-diameter gold-coated sapphire sphere exerts a force whose gradient $\partial F / \partial z$ contributes to the oscillator's effective torsion constant. Capacitor plates below the oscillator are used to drive it at resonance, while the height of the sphere is varied harmonically with time. The force $F(z)$ between sphere and oscillator plate is derived from the variation in resonant frequency as a function of $z$. The sphere-plate separation was varied from 160 to $750 \mathrm{~nm}$, measuring forces with relative error varying from $0.19 \%$ at $160 \mathrm{~nm}$ to $9.0 \%$ at $750 \mathrm{~nm}$

produced extremely reproducible results, with Yukawa force limit certainty apparently limited only by uncertainty in Casimir force calculation.

Dimopoulos and Geraci (2003) in consultation with Mark Kasevich and others, have proposed a probe of submicron-range forces using interferometry of Bose-Einstein condensed atoms. This approach, illustrated in Fig. 16, would load two laser-trap regions at differing distance from a source mass with Bose-Einstein condensates of well-defined initial relative phase. The difference in phase evolution rate of the two condensates would yield a measure of the different potentials in which they sit. After an appropriate interval the condensates would be combined, and their relative phases read out with a fluorescence technique. The contribution to the relative phase shift due to the source mass potential would 


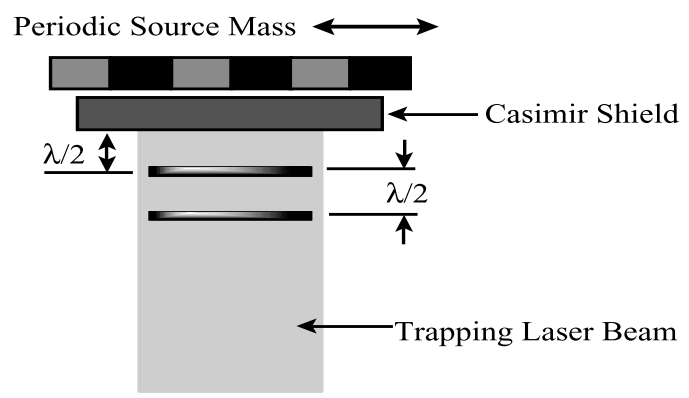

Fig. 16 The experimental concept of Dimopoulos and Geraci for probing ultra-short distances with an atom interferometry technique. On one side of a gold fixed Casimir shield, an array of tiny source masses of alternating density would move laterally as indicated. On the other side of the gold shield, BEC atom clusters would be trapped in two potential wells a distance $\lambda / 2$ apart in a standing wave cavity formed by reflection of a $\lambda=840 \mathrm{~nm}$ laser from the shield. The BEC clusters would be loaded with an initial well-defined relative phase, whose evolution in time would reflect the different potentials in which they sit. Comparison of relative phase evolution rates for different source mass positions then serves to separate the source-mass-related contribution to the relative potentials at the two regions from the contribution from Casimir effects

be isolated from that due to Casimir and other potentials, by modulating the source mass position.

Measurements of neutron phenomena have been used to put limits on a new Yukawa force; see (Nesvizhevsky et al. 2008; Nesvizhevsky and Protasov 2004) and references therein. Such studies include analyses of the dependence of neutron scattering length on target atomic number, and measurements of quantized neutron energy levels in the Earth's gravitational field. While these limits on $\alpha$ are weak, they extend to ranges below $10^{-11}$ meters. New techniques that could improve such limits using neutrons have been proposed by Greene and Gudkov (2007), Nesvizhevsky et al. (2008) and others. A limit on $\alpha$ extending to extremely short ranges comes from analysis by Nesvizhevsky and Protasov (2004) of data on transition frequencies in antiprotonic atoms $\left(\bar{p}^{3} \mathrm{He}^{+}\right.$and $\left.\bar{p}^{4} \mathrm{He}^{+}\right)$. This limit derives from the fact that a new Yukawa interaction between the antiproton and its partner nucleus, with range $\lambda$ greater than the extremely small Bohr radius of the antiprotonic atom, would change the apparent value of the electric binding energy of the system, with a corresponding change in transition frequencies. A $1 \sigma$ limit $|\alpha| \leq 1.3 \times 10^{28}$ is obtained, valid for ranges down to $10^{-13} \mathrm{~m}$ or less.

Constraints in $\alpha-\lambda$ space from Casimir, neutron, and atomic studies are shown in Fig. 17.

\section{Theoretical Ideas Suggesting Inverse Square Law Violation}

A wide variety of theoretical ideas suggest a breakdown of the gravitational inverse square law in experimentally accessible regions. Such ideas as of 2003 are reviewed by Adelberger, Heckel and Nelson (AHN) (Adelberger et al. 2003), while more recent ideas (chameleons, unparticles, "fat" gravitons) and their constraints are discussed by Adelberger and colleagues in Adelberger et al. (2009). Here we outline some of these ideas.

One much-referenced idea is the suggestion (Arkani-Hamed et al. 1998) (ADD) that the hierarchy problem presented by the vast difference between Planck and electroweak energy scales could be resolved if there are large extra compact dimensions in which gravitons (but not Standard Model particles) propagate. In the ADD scenario with n equal size extra 


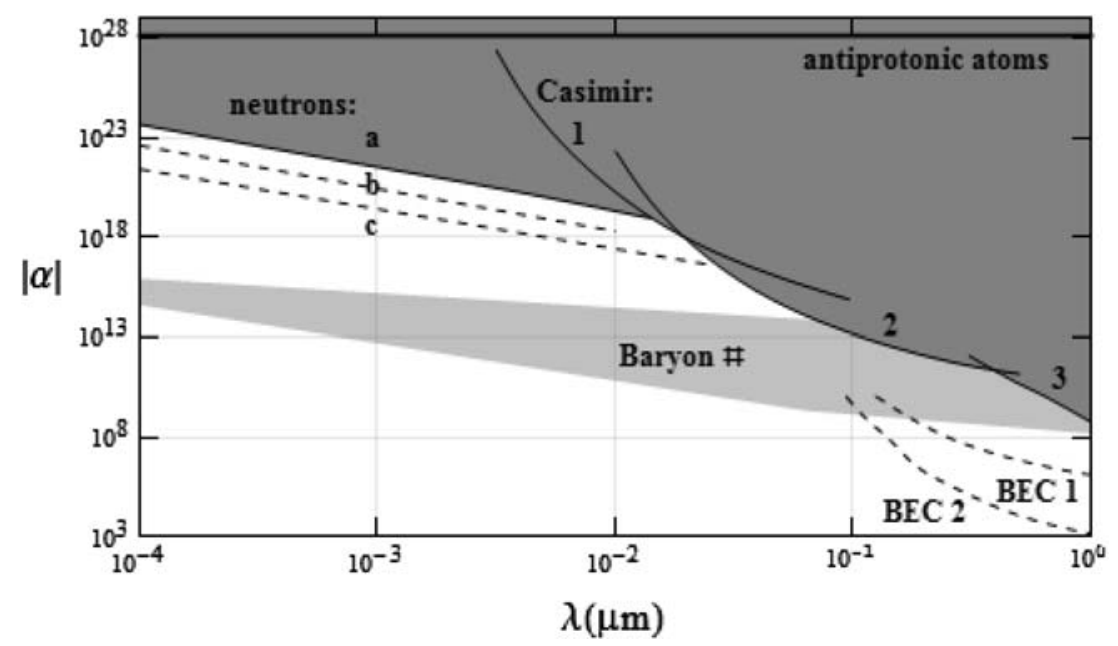

Fig. 17 Constraints in $\alpha-\lambda$ parameter space based on Casimir, neutron, and antiprotonic atom experiments. Lines 1 and 2 are 95\% confidence limits derived by Mostepanenko et al. (2008) from the Casimir experiments of Harris and Chen (2000) and Decca et al. (2005) respectively. Line 3 is based on the Casimir experiment of Lamoreaux (1997). Line a is a constraint obtained by Nesvizhevsky et al. (2008) from neutron scattering data; lines $\mathrm{b}$ and $\mathrm{c}$ are respectively constraint improvements that might be achieved using neutron experiments suggested by Nesvizhevsky et al. (2008) and Greene and Gudkov (2007). The antiprotonic line is the constraint from antiprotonic atom transition frequencies. A region is displayed in which a Baryon gauge particle discussed by Dimopoulos and Geraci (2003) might manifest itself, while dashed lines BEC 1 and 2 indicate potential sensitivity of the experimental technique these authors suggest

dimensions of radius $R$, the fundamental energy scale for gravity, $M_{*}$, is comparable to the electroweak scale $m_{E W}$ and is related to the Planck mass by $M_{P l}^{2} \sim M_{*}^{2+n} R^{n}$. Putting $M_{*} \sim m_{E W}$ then yields:

$$
R \sim 10^{\frac{30}{n}-17} \mathrm{~cm} \times\left(\frac{1 \mathrm{TeV}}{m_{E W}}\right)^{1+\frac{2}{n}}
$$

In this scheme, Kaluza-Klein (KK) excitations in the extra dimensions give rise to an ISL-violating interaction energy $V(r)=-\alpha \frac{G m_{1} m_{2}}{r} e^{-r / \lambda}$, where $\lambda \sim R$ and $\alpha$ (for $n=2$ ) is between 3 and $\sim 20$, depending on the topology of the extra dimensions (Kehagias and Sfetsos 2000). Taking $m_{E W}=1 \mathrm{TeV}$ and $n=1$ leads to $R \sim 10^{13} \mathrm{~cm}$, which is empirically excluded, while for $n \geq 3, R$ would be less than a nanometer. For $n=2, R$ would be in an experimentally accessible range, $\sim 100 \mu \mathrm{m}-1 \mathrm{~mm}$. $R$ in this range is excluded by present experiments (Kapner et al. 2007), and as the AHN review paper notes, is also excluded by arguments based on neutrino emission from supernova 1987A, big-bang nucleosynthesis, and non-observation of a diffuse background of cosmological gamma rays from the decay of KK modes. However, AHN discuss other extra-dimension scenarios which can still viably suggest ISLV in a similar distance range, while addressing the hierarchy problem. These scenarios include a model constructed with one large extra dimension $(\sim 1 \mathrm{~mm})$ along with several much smaller extra dimensions.

Other possible sources of ISLV are various moduli which arise in superstring theory. Originally massless, such moduli can acquire mass through a variety of possible mechanisms, on various mass scales. One of these moduli is the dilaton, which plays a role in controlling the strength of all interactions. Its mass is unknown, but it would 


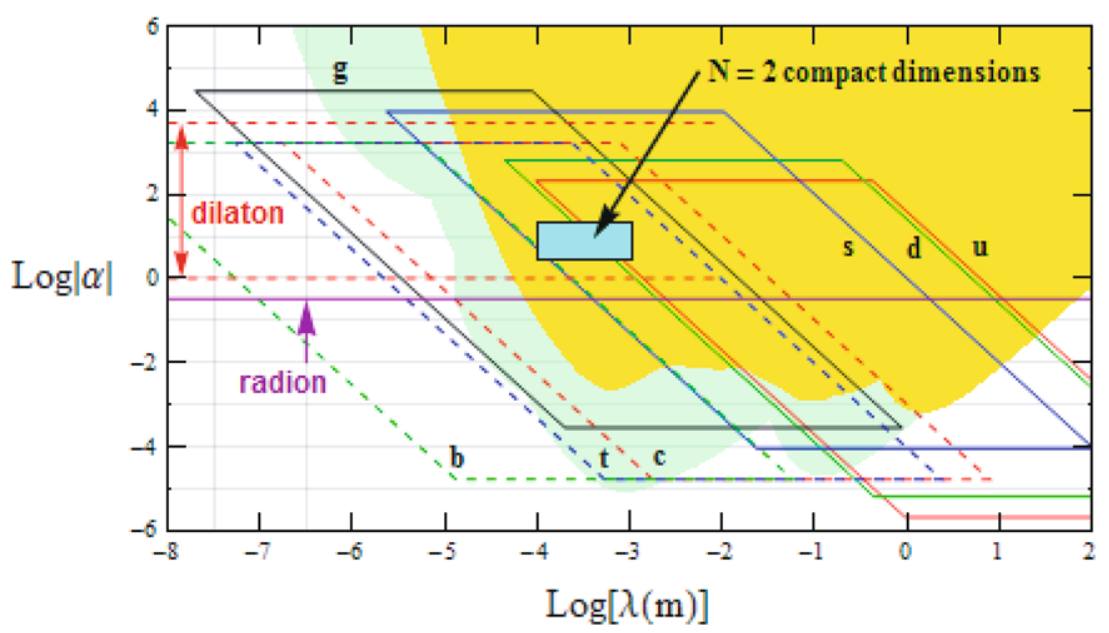

Fig. 18 Theoretical suggestions of regions in $\alpha-\lambda$ parameter space where anomalous forces might be revealed. Regions in which Dimopoulos and Geraci (2003) suggest moduli arising in superstring theory might manifest themselves are roughly bounded by parallelograms labeled according to the particle to which the modulus would couple: $\mathrm{u}, \mathrm{d}, \mathrm{s}, \mathrm{c}$, $\mathrm{t}$, or b quark, or gluon $(\mathrm{g})$. A region in which two equal-size extra dimensions might be manifested in the ADD scenario (Kehagias and Sfetsos 2000) is indicated, along with regions that might manifest a dilaton (Kaplan and Wise 2000) or radion (Antoniadis et al. 1998). The two shaded regions indicate best current experimental limits and potentially improved limits on an interaction of the form of (1)

be expected to produce an interaction between atoms with strength $\alpha$ relative to gravity in a range 1 to $\sim 5000$ (Kaplan and Wise 2000). In addition there may be a number of other moduli, with couplings similar to but more specific than that of the dilaton. A possibility explored by Dimopoulos and collaborators (Dimopoulos and Giudice 1996; Dimopoulos and Geraci 2003) is that such moduli acquire mass as a result of supersymmetry breaking at a low energy scale $F$, acquiring mass proportional to $\sim F / M_{P l}$, with $\sqrt{F}$ in a range $10 \mathrm{TeV}$ to $2000 \mathrm{TeV}$. These moduli include a gluon modulus that would couple only to Standard Model gluons, and Yukawa moduli that would determine quark and lepton masses and, if sufficiently light, mediate additional interactions. Another possibility is one or more "radions", which can modify effective couplings through their role in fixing the size of compact extra dimensions. A radion would mediate an ISL-violating force with strength $\sim 1 / 3$ of gravity (Antoniadis et al. 1998). Yet another possibility is a bulk gauge particle, such as a baryon gauge particle that couples to baryon number but is free to move off the brane in an extra-dimensional scenario. This possibility is of particular interest to Dimopoulos and his collaborators, because it might manifest itself as ISLV in a small- $\lambda$, large- $\alpha$ region in $\alpha-\lambda$ parameter space which could be accessible with the novel BEC experimental technique described in the preceding section and in (Dimopoulos and Geraci 2003). Figure 18 displays some of the regions in $\alpha-\lambda$ parameter space in which theories discussed above suggest possible inverse square law breakdown.

Up to this point, this paper has considered only constraints on anomalous interactions of the Yukawa form represented in (1). However inverse square law deviations of power law form could arise from the exchange of multiple bosons. The best limits on such interactions come from the work of the UW Eöt-Wash group (Adelberger et al. 2007). Assuming an 
interaction of the form

$$
V_{n}=-\frac{G m_{1} m_{2}}{r}\left(1+\beta_{n}\left(\frac{1 m m}{r}\right)^{n-1}\right)
$$

$1 \sigma$ limits on $\beta_{n}$ for $n=2,3,4$ and 5 are found to be respectively $4.5 \times 10^{-4}, 1.3 \times 10^{-4}$, $4.9 \times 10^{-5}$, and $1.5 \times 10^{-5}$.

Acknowledgements The authors' work described here is supported by NSF grants PHY-0701707 and PHY-0701923, and in part by the Pacific Northwest National Laboratory.

\section{References}

E.G. Adelberger, B.R. Heckel, A.E. Nelson, Ann. Rev. Nucl. Part. Sci. 53 77, (2003)

E.G. Adelberger, B.R. Heckel, S. Hoedl, C.D. Hoyle, A. Upadhye, Phys. Rev. Lett. 98, 131104 (2007)

E.G. Adelberger, J.H. Gundlach, B.R. Heckel, S. Hoedl, S. Schlamminger, Prog. Part. Nucl. Phys. 72, 102 (2009)

I. Antoniadis, S. Dimopoulos, G. Dvali, Nucl. Phys. B 516, 70 (1998)

N. Arkani-Hamed, S. Dimopoulos, G. Dvali, Phys. Lett. B 429, 263 (1998)

P.E. Boynton, R.M. Bonicalzi, A.M. Kalet, A.M. Kleczewski, J.K. Lingwood, K.J. McKenney, M.W. Moore, J.H. Steffen, E.C. Berg, W.D. Cross, R.D. Newman, R.E. Gephart, New Astron. Rev. 51, 334 (2007)

H.A. Chan, M.V. Moody, H.J. Paik, Phys. Rev. Lett. 49, 1745 (1982)

Y.T. Chen, A.H. Cook, A.J.F. Metherell, Proc. R. Soc. Lond. A 394, 47 (1984)

R.S. Decca, D. López, H.B. Chan, E. Fischbach, D.E. Krause, C.R. Jamell, Phys. Rev. Lett. 94, 240401 (2005)

S. Dimopoulos, G.F. Giudice, Phys. Lett. B 379, 105 (1996)

S. Dimopoulos, A.A. Geraci, Phys. Rev. D 68, 124021 (2003)

A.A. Geraci, S.J. Smullin, D.M. Weld, J. Chiaverini, A. Kapitulnik, Phys. Rev. D 78, 022002 (2008)

J.M. Goodkind, P.V. Czipott, A.P. Mills, M. Murakami, P.M. Platzman, C.W. Young, D.M. Zuckerman, Phys. Rev. D 47, 1290 (1993)

G.L. Greene, V. Gudkov, Phys. Rev. C 75, 015501 (2007)

G.D. Hammond, C.C. Speake, A.J. Matthews, E. Rocco, F. Peña-Arellano, Rev. Sci. Instrum. 79, 025103 (2008)

B.W. Harris, F. Chen, U. Mohideen, Phys. Rev. A 62, 052109 (2000)

J.K. Hoskins, R.D. Newman, R. Spero, J. Schultz, Phys. Rev. D 32, 3084 (1985)

C.D. Hoyle, U. Schmidt, B.R. Heckel, E.G. Adelberger, J.H. Gundlach, D.J. Kapner, H.E. Swanson, Phys. Rev. Lett. 86, 1418 (2001)

C.D. Hoyle, D.J. Kapner, B.R. Heckel, E.G. Adelberger, J.H. Gundlach, U. Schmidt, H.E. Swanson, Phys. Rev. D 70, 042004 (2004)

D.B. Kaplan, M.B. Wise, J. High Energy Phys. (2000)

D.J. Kapner, T.S. Cook, E.G. Adelberger, J.H. Gundlach, B.R. Heckel, C.D. Hoyle, H.E. Swanson, Phys. Rev. Lett. 98, 021101 (2007)

A. Kehagias, K. Sfetsos, Phys. Lett. B 472, 39 (2000)

S.K. Lamoreaux, Phys. Rev. Lett. 78, 5 (1997)

D.R. Long, Phys. Rev. D 9, 850 (1974)

D.R. Long, Nature 260, 417 (1976)

D.R. Long, Nuovo Cimento B 55, 252 (1980)

J.C. Long, H.W. Chan, A.B. Churnside, E.A. Gulbis, M.C.M. Varney, J.C. Price, Nature 421, 922 (2003)

M.V. Moody, H.J. Paik, Phys. Rev. Lett. 70, 1195 (1993)

M.W. Moore, Ph.D. Thesis, UMI \#9964273, University of Washington (2000)

V.M. Mostepanenko, R.S. Decca, E. Fischbach, G.L. Klimchitskaya, D.E. Krause, D. López, J. Phys. A: Math. Theor. 41, 164054 (2008)

V.V. Nesvizhevsky, K.V. Protasov, Class. Quantum Gravity 21, 4557 (2004)

V.V. Nesvizhevsky, G. Pignol, K.V. Protasov, Phys. Rev. D 77, 034020 (2008)

Y. Ogawa, K. Tsubono, H. Hirakawa, Phys. Rev. D 26, 729 (1982)

V.I. Panov, V.N. Frontov, Sov. Phys. JETP 50, 852 (1979)

R. Spero, J.K. Hoskins, R. Newman, J. Pellam, J. Schultz, Phys. Rev. Lett. 44, 1645 (1980)

L.-C. Tu, S.-G. Guan, J. Luo, C.-G. Shao, L.-X. Liu, Phys. Rev. Lett. 98, 201101 (2007)

D.M. Weld, J. Xia, B. Cabrera, A. Kapitulnik, Phys. Rev. D 77, 062006 (2008) 